\title{
Inhibition of nitric oxide and prostaglandin E2 production by pyrrolylated-chalcones: synthesis, biological activity, crystal structure analysis, and molecular docking studies
}

\begin{abstract}
In search of potent anti-inflammatory agents, twenty-four chalcone derivatives including seven new compounds (13 - 17, 21 and 23) containing pyrrole moiety were designed, synthesized, and assessed for their nitric oxide (NO) and prostaglandin E2 (PGE2) suppression ability on IFN- $\gamma /$ LPS-induced RAW 264.7 macrophage cells. Results showed that none of the synthesized compounds were PAINS-associated molecules, with 3-(2,5-dimethoxyphenyl)-1(1H-pyrrol-2-yl)-prop-2-en-1-one (compound 16) exhibiting remarkable inhibition activity towards PGE2 and NO production with IC50 values of $0.5 \pm 1.5 \mu \mathrm{M}$ and $12.1 \pm 1.5 \mu \mathrm{M}$, respectively. Physicochemical and ADMET studies showed that majority of the compounds obey to Lipinski's rule of five (RO5) having high blood brain barrier (BBB) penetration, human intestinal absorption (HIA), P- glycoprotein ( $\mathrm{PgP}$ ) inhibition and plasma binding protein (PPB) inhibition. The obtained atomic coordinates for the single-crystal XRD of 16 were then applied in a molecular docking simulation, and compound 16 was found to participate in a number of important binding interactions in the binding sites of ERK and mPGES-1. Based on these results, we have observed the potential of compound 16 as a new hit anti-inflammatory agent, and these findings could serve as a basis for further studies on its mechanism of action.
\end{abstract}

